\title{
Risultati di alcune ricerche sulla serie gessoso solfifera siciliana
}

\author{
1. FrascivigLia
}

Ricevuto il 5 Febbraio 1962

\section{PRemessi.}

Nel corso del rilevamento geologico sistematico necessario per la seconda edizione della Carta geologica della Sicilia, ho rilevato buona parte della Sicilia centro meridionale, quella, ció̀, dove è maggiormente sviluppata la serie gessoso solfifera. Nelle zone di interesse minerario, quale base topografica ho adottato le levate originali alla scala di $1: 5.000$, compilate dall'I. G. M. nell'ante-guerra, su commissione dell'allora esistente Consorzio Solfifero.

Il lavoro, oltre che per la redazione della Carta geologica, doveva servire all'Ente Zolfi Italiani (E. Z. I.) quale base di orientamento per la ricerca solfifera. Ho dovuto, perciò, dedicare molta attenzione alla serie gessoso solfitera, specialmente alla posizione reciproca attuale dei suoi diversi lembi, in conseguenza delle vicende tettoniche subite dalla serie dopo il suo deposito.

Durante questo lavoro l'E.Z. I. mi ha sempre tenuto al corrente dei rilevamenti geologici di dettaglio eseguiti dagli operatori alle sue dirette dipendenze, nonché dei risultati delle ricerche geofisiche condotte da diverse Società.

In questa nota mi occuperò di due zone: Monte Stretto, nella tavoletta "Imera " (268 III SE), e la conca di Trabia Tallarita nella tavoletta "Ravanusa" (272 IV NO), da me studiate in dettaglio, poiché in esse sono stati eseguiti da parte della Compagnie Générale de Géophysique (C.G.G.) dei sondaggi elettrici (S. E.), e delle trivellazioni esplorative.

Per potere valutare a priori la possibilità d'impiego del metodo dei S. E. nel particolare problema proposto, e interpretare correttamente 
le curve di campagna, sono stati messi a disposizione della C. G. G. i rilevamenti geologici di dettaglio, i profili stratigrafici delle trivellazioni già eseguite, nonché tutti i dati ricavabili dalle vecchie coltivazioni minerarie e da quelle in atto.

\section{Zona di Monte StretTo.}

In questa zona l'affioramento della serie gessoso solfifera si presenta sotto forma di un'omega, costituito dall'affioramento rettilineo di Gessolungo, diretto NO-SE con immersione verso SO e pendenze variabili che arrivano fino a $60^{\circ}$ all'estremita occidentale, seguito ad oriente dall'affioramento di Monte Stretto, piegato a ferro di eavallo e rotto da numerose piccole faglie trasversali. In questo tratto le pendenze convergono verso il centro del bacino, ma variano da punto a punto: si hanno strati mediamente inclinati vicino la $\mathrm{C}$. Stretto; fortemente inclinati, circa $70^{\circ}$, nella solfara Saponaro-Garibaldi, all'estremitì orientale dell'affioramento; sub verticali nella ricerea Pizzuto, sul lato ocridentale dell'omega.

Un torrente, probabilmente impiantato sopra una laglia, separa l'affioramento di Gessolungo dalla zona dove sono ubicate le miniere di Giangiana ed altre limitrofe, oggi abbandonate.

Le coltivazioni minerarie hanno interessato maggiormente il lato orientale dell'omega, dove sono tuttora visibili numerosi calcaroni e grossi depositi di rosticci. All'epoca del mio rilevamento, oltre ad una sezione della miniera Saponaro, erano attive la ricerca Pizzuto e un'altra più a Sud, dove si cercava l'eventuale prosecuzione verso Nord di un banco di minerale, già cultivato nella zona Juncio.

L'interno del bacino di Monte Stretto ì colmato dalla formazione di quelle particolari argille eterogenee, di aspetto brecciato, oggichiamate " argille brecciate "; nome dato loro da Ogniben (1953, 1954), che corrispondono alle "arsille scagliose " di Beneo (1949a, 19-19b, 1950). In un traversobanco della ricerea Pizzuto ho raccolto personalmente in uno strato a tetto della serie gessoso solfifera dei campioi di un'argilla azzurrognola contenente un'abbondante microfauna di foraminiferi costituenti un'associazione tipicamente tortoniana. Sul lato orientale, fra gessi e trubi, si trova uno straterello di un calcare biancastro con piccoli macrofossili ben conservati, in tutto simile a quello descritto da Baldacei (1886) come facente parte degli strati a congerie (Francaviglia e Jacobacci, 1953). 
La mia interpretazione della struttura di Monte Stretto ì stata illustrata nella nota suindieati, alla quale rimando per maggiori dettagli. Per analogia a quanto avevo potuto constatare in altri posti, e per quanto avevo potuto vedere nei sotterranei delle vecehie coltivazioni, se accessibili, e dedurre dai piani di miniera, lıo considerato questa strutturi come una pseudosinclinale, originata dall'arricciamento di un lembo non molto largo di serie gessoso solfitera, stacato per faglia dal più grosso bloceo sul quale sono impiantate le miniere Giangiana, Giordano (oggi esaurite) ed altre minori, come si vede nel blocco-diagramma della Fig. 1.

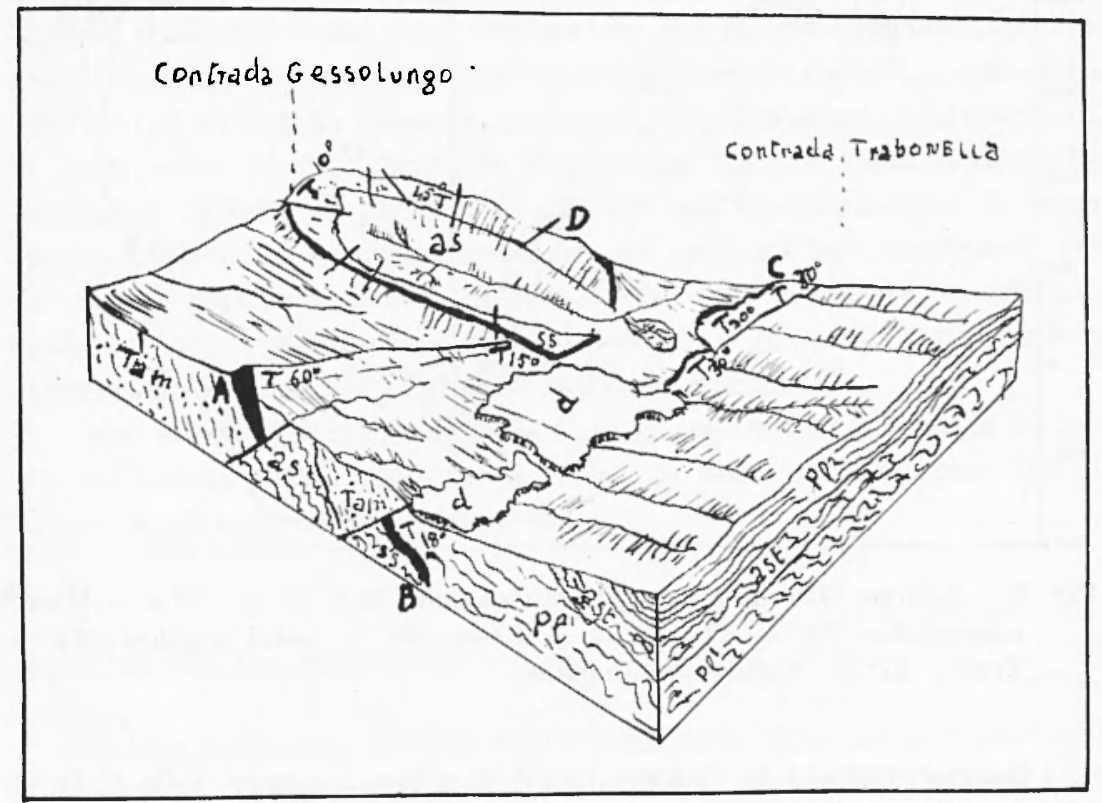

Fig. 1 - Interpretazione strutturale degli affioramenti solfiferi a Gessolungo (A); Giangiana (B); Trabonella (C) e Monte Stretto (D).

Tam = Marne mioceniche; $S S=$ Serie solffera; $P l^{1}=$ Trubi; $I^{\prime} l^{2}=$ Argille di tipo piacenziano; $a s=$ Argille brecciate; $a s t$ " Argille brecciate con lembi di trubi; $d=$ Rosticci.

Questa mia interpretazione non è stata accettata dai geologi alle dirette dipendenze dell'E. Z. I., i quali, invece, hanno sostenuto l'ipotesi di un bacino sinclinatico.

Per una migliore conoscenza della situazione tettonica venne eseguito in foro di sonda, Juncio sociale 1 ; sono stati attraversati $60 \mathrm{~m}$ di argille di ricoprimento, poi fino a circa $88 \mathrm{~m}$ una formazione analoga a. 
quella incontrata nella tavoletta "S. Caterina Villarmosa " (268 IV NO) che ho chiamato "argilla con gessi ", perchè formata da banchi argillosi riechi di sottilissimi letti di gesso (Francaviglia 1953), alla quale ha. fatto seguito una lente di salgemma con sottili intercalazioni argillose e gessose, ed inflne, a circa $290 \mathrm{~m}$, un po' di gesso seguito da marne tripolacee.

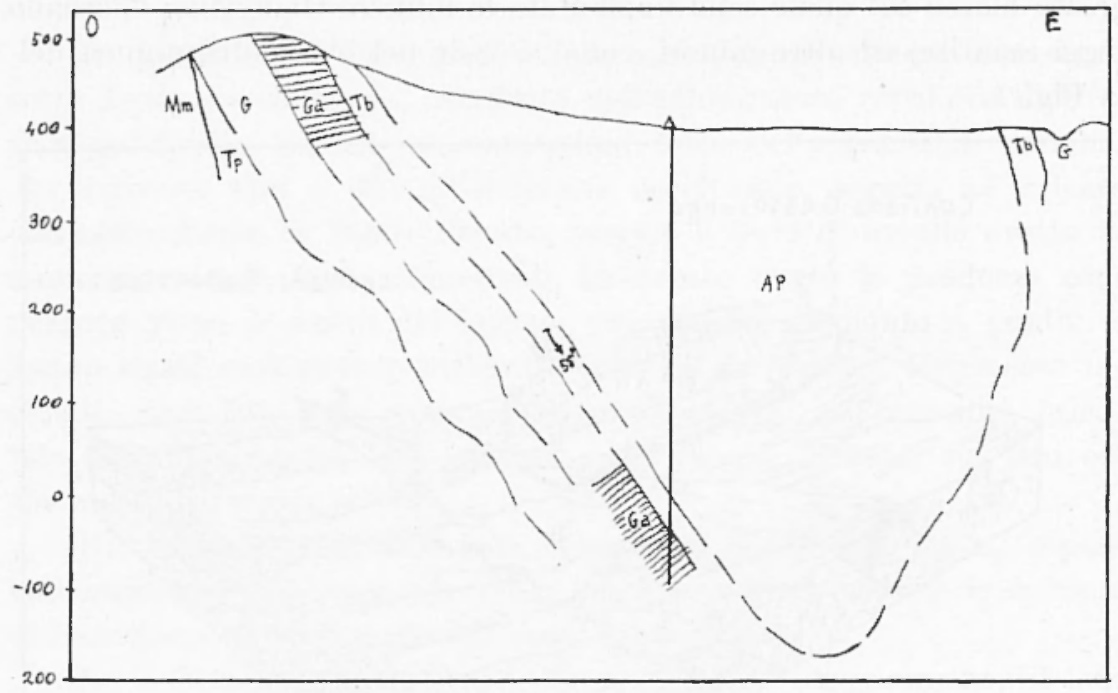

Fig. 2 - Sezione O.E di Monte Stretto secondo la C. G. G.; $M L n=\operatorname{Marn} \theta$ mioceniche; $T_{p}=$ Tripoli; $G=$ Gessi; $G a=$ Gessi argillosi; $T b=$ Trubi; $A P=$ Argille pnddingoidi.

Questo risultato ha indotto l'E. \%. I. a fare eseguire dalla C. G. G. alcuni sondaggi elettrici; ne sono stati eseguiti quattro, il primo dei quali ubicato accanto al foro di sonda Juncio sociale 1.

I quattro diagrammi, astrazione fatta del tratto iniziale influenzato dalle condizioni di superficie, sono riferibili ad un complesso a due strati, con quello superiore a resistività più bassa dell'inferiore; manca qualsiasi accenno a ridiscesa nella parte terminale delle curve, per quanto le stese elettrodiche siano state portate fino ad interessare una profondità di $500 \mathrm{~m}$. Tall'esame di questi diagrummi si dovrebbe dedurre che sotto una copertura a bassa resistività dovrebbe esistere un substrato a resistività parecchio più alta, estendentesi molto in profondità; ciò è in assoluto contrasto sia con i lavori minerari, sia col risultato del foro di sonda Juncio sociale 1. 
La C. G. G. (1954) ha però concluso che "i diagrammi hanno inizio con una resistività da 1,5 a $2 \mathrm{ohm}$, e la resistività aumenta progressivamente: ne deduciamo la presenza della serie solfifera " in contrasto con tutti i risultati fino allora aequisiti.

Prima della stesura della relazione definitiya, era stato portato a termine il foro di sonda Juncio sociale 2 , in vicinanza del S.E. n. 4 , che secondo un precedente rapporto provvisorio, avrebbe doruto incontrare la serie gessoso solfifera a circa $200-250 \mathrm{~m}$ di profondità. I risultati del foro non hanno confermato questa previsione e nemmeno l'ipotesi dell'esistenza di un bacino sinclinalico. Infatti, dopo $395 \mathrm{~m}$ di argille brecciate sono stati incontrati dei trubi, seguiti da una formazione che la C. G. G. indiea come gesso argilloso in una sezione allegata alla relazione (Fig. 2), come argilla sarmaziana nel diagramma del S.E., e come serie solfifera nel testo della relazione, indicando così l'imbarazzo dell'estensore', il quale cerca di giustifieare la discrepanza fra previsioni e risultato, invocando l'effetto di parete eil fatto che nell'interpretazione "le curve sperimentali sono confrontate con abachi calcolati per condizioni (per es. deboli pendenze) molto differenti dal caso presente ", e conclude scrivendo che "per lo studio di bacini stretti e profondi la prospezione elettrica deve essere applicata con molta discrezione"

Non si comprende come mai la C. G. G., che era a conoscenza di questa condizioni, abbia applicato lo stesso un metodo di indagine difficilmente applicabile al caso proposto.

\section{Zona di Trabia Tallariti.}

È una delle zone dove le coltivazioni sono state iniziate da secoli. Si ha qui un bacino grossolanamente ellittico, contomato da tutti i lati da affioramenti della serie gessoso solfifera, e attraversato in direzione $\mathrm{N}$-S dall'Imera meridionale $\mathrm{e}$ in direzione quasi E-O dal suo affluente di destra Gibbesi.

Lungo i bordi del bacino gli affioramenti sono fortemente inclinati, specialmente a Roca Messana (bordo occidentale) e a Serra Perciata (bordo orientale), o addirittura in contropendenza come a Serra Montagna; però, nella parte settentrionale di Serra del Bosco le pendenze sono minori e si riducono a pochi gradi.

La composizione della serie gessoso solfifera non è la stessa lungo tutto l'arco degli affioramenti; in generale i gessi hanno la preponderanza sui calcari, i quali sono di spessore molto ridotto negli affioramenti della. 
Solfarella, lato orientale del bacino, e mancano del tutto nella parte settentrionale. Le vicende tettoniche hanno smembrato la serie gessoso solfifera, riducendola a volte a lembi piccoli e piccolissimi, con fenomeni di sovrascorrimento, come quelli osservabili a Poggio Conte Bosco.

Le pendenze degli affioramenti convergono verso l'interno del bacino, che, a prima vista, può essere considerato come una conca sinclinalica; ma un attento esame delle condizioni locali non consente di accettare per intero questa ipotesi.

Sulla serie gessoso solfifera si adagiano i trubi, a volte con marcata discordanza angolare, con una potenza di una quindicina di metri, ai quali segue una formazione argillosa formata principalmente da argille brecciate, considerate da Baldacci (1886) come argille tortoniane antoctone, e da Berhmann (1938) come una falda di ricoprimento, venuta in posto per un fenomeno speciale di diapirismo. Le argille breceiate hanno invaso il bacino poco dopo l'inizio della sedimentazione dei trubi; infatti, quelle provenienti dalla discarica della galleria ferroviaria che passa sotto la strada da Sommatino a Riesi sono costituite da un impasto di argille con microfauna tortoniana e argille decisamente plioceniche; queste argille inglobano esotici di rocee diverse, a volte di notevole dimensione, come lo spuntone di arenaria a glauconite, che si trova sulla Serra Palladio, a NE della stazione ferroviaria.

Ma le argille brecciate si trovano anche nei terreni sottostanti alla serie gessoso solfifera. i possibile vederle sul rovescio di Serra Palladio; a SO del ponte ferroviario rotto si trovano estesi banchi di arenaria quarzosa e grandi blocehi di un calcare marnoso biancastro, di aspetto simile ai trubi, ma racchiudente una microfauna di foraminieri riferibili all'Oligocene, secondo le determinazioni di Lipparini. Nell'incisione valilva del Gibbesi, a NO di Rocca Messana, si ritrovano questi stessi calcari, in strati inclusi in una formazione argillosa con microfauna oligo-miocenica.

La dorsale di S. Anna, nel centro del bacino, è formata da arerille con mierofauna di foraminiferi tortoniani, ricoperte da un banco di conglomerato a piccoli elementi di rocce diverse, analogo, salvo che per la dimensione dei ciottoli, a quelli che ho incontrato da $\mathrm{S}$. Caterina Villarmosa fino al mare. Pì̀ a Yord, nella contrada Ulivella, sono presenti dei gessi in posizione anormale; infatti essi poggiano in assoluta discordanza sui congolmerati di cui sopra.

Ho seguito il rilevamento geologico di dettaglio di questa zona nell'estate del 1949 , visitando tutte le gallerie accessibili del sotterraneo della miniera, ed ho potuto avere a disposizione $\mathrm{i}$ piani delle vecchie coltivazioni, alcuni dei quali rimontavano alla meti del secolo scorso. 
Nella mia relazione all'E.Z. I. (Francaviglia, 1949) ho affacciato l'ipotesi che nell'interno del bacino potesse mancare la continuità della serie gessoso solfifera; ipotesi che ho ribadito nella relazione sul rilevamento geologico della tavoletta "Ravanusa" (Francaviglia 1953), nella quale ho detto che, a mio parere, il bacino di Trabia Tallarita doveva essere considerato come una pseudosinclinale, formata da un accostamento casuale di lembi diversi di serie gessoso solfifera, e che perciò non era probabile la presenza di questa verso il centro del bacino, Fig. 3.

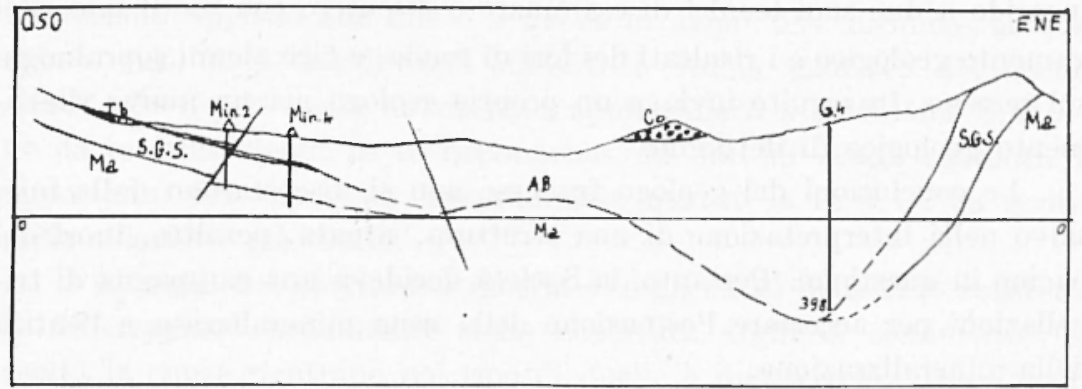

Fig. 3 - Sezione Mintina Solfarella, secondo Francaviglia $M a=$ Miocene argilloso; S. G.S. = Serie gessoso solffera; $A B=$ Argille brecciate; Co $=$ Conglomerato; $\operatorname{Min}=$ Fori di sonda esistenti; $G 4=$ Foro di controllo.

Nella citata relazione all'E.Z. I. (1949), riferendomi alla possibilità d’impiego dei metodi geofisici per la ricerca solfifera nel bacino, scrivevo che la geoelettrica in corrente continua (misure della resistività (lel suolo, cioè S. E.) aveva delle limitazioni di impiego dipendenti dalla morfologia del terreno e dal motivo tettonico delle zone da investigare, nonchè dalle condizioni del terreno di copertura, e che perciò il metodo era applicabile con buone probabilità di successo soltanto in tre piccolissimi tratti del bacino; consigliavo l'applicazione del metodo sisnico.

Personalmente, nel 1919, ho eseguito dei S.E. in due delle zone indicate, scartando la terza in base a considerazioni geologiche. In una di queste, e precisamente a Piano Pinta, venne successivamente eseguita una trivellazione esplorativa, che diede un risultato positivo dal punto di vista geologico, in quanto ha incontrato la serie gessoso solfifera alla profondità prevista, ma negativo dal punto di vista minerario, perchè venne attraversata una zona già coltivata. Questo risultato ha indotto il mio collega $\Lambda$. Manfredini (1950) e successivamente me (1951) a pubblicare i risultati dei nostri esperimenti sulla possibilità dell'impiego 
del metodo dei S.E., nella ricerca della serie gessoso solfifera, che avevamo condotto assieme in altre zone.

In un secondo tempo l'E. $/$. . I. decise di fare eseguire una serie di fori di sonda nella parte occidentale del bacino; venne rivelata cosi l'esistenza nel sottosuolo della serie gessoso solfifera più o meno mineralizzata, e si rilevò qualche elemento in favore dell'ipotesi della non esistenza della serie stessa nella parte centrale del bacino.

La presenza della mineralizzazione destò l'interesse di una Società mineraria francese, la Compagnie des Mines de Huaron, la quale diede incarido a due suoi tecnici di esaminare e discutere con me il mio rilevamento geologico e i risultati dei fori di sonda, e fare alcuni sopraluoghi sul terreno. In seguito inviava un proprio geologo per un nuovo rilevamento geologico di dettaglio.

Le conclusioni del geologo francese non si diseostarono dalle mie, salvo nella interpretazione di una struttura, situata, peraltro, fuori del bacino in questione. Pertanto, la Società decideva una campagna di trivellazioni per accertare l'estensione della zona mineralizzata e l'entità della mineralizzazione.

Purtroppo i risultati furono minerariamente sconfortanti per la scarsezza della mineralizzazione e per la sua discontinuità. Sono serviti, però, a mettere in evidenza alcuni fatti di notevole importanza, e cioè:

1) la pendenza degli strati della serie gessoso solfifera, molto forte sugli affioramenti occidentali, si addolcisce verso il centro del bacino, dove le carote hanno permesso di misurare una pendenza di soli $10^{n}$;

2) la potenza della serie gessoso solfifera diminuisce verso valle, e la serie comincia ad essere molto tormentata, come lo dimostra la presenza di brecce di argille marnose e calcari solfiferi rilevate nel foro n. 12 bis:

3) a valle del foro sudetto esiste certamente un disturbo tettonico; infatti mentre in detto foro la serie gessoso solfifera è stata incontrata a $115 \mathrm{~m}$ di profondità, non è stata incontrata nel foro più a valle, il n. 13 spinto a $396 \mathrm{~m}$ di profondità;

4) le argille dell'ultima carota del foro n. 13, studiate dalla Sig.na Dr. Tamajo del Centro Sperimentale per le Industrie Minerarie di Palermo, contengono una microfauna di foraminiferi elveziano-tortoniana, analoga a questa delle argille della dorsale di S. Anna. 
Questi risultati hamno indotto il geologo francese ad ammettere nella sua relazione (Jaoul, 1952) che la serie gessoso solfifera potesse non esistere verso il centro del bacino. In base a questa relazione, la Società rinunciava a proseguire le ricerche.

L'ipotesi della non continuità della serie gessoso solfifera non ha incontrato il favore dei geologi alle dirette dipendenze dell'E. Z. I., e l'Ente, nel quadro dello studio sistematico di tutti i bacini solfiferi della Sicilia, ha creduto opportuno di fare eseguire un nuovo rilevamento geologico di dettaglio, affidandolo ad uno di essi, il quale è pervenuto a conclusioni opposte alle mie e a quelle di Jaoul. Per dirimere la questione, l' E. Z. I. decideva di affidare lo studio geofisico del bacino alla C. G. G., la quale ha ritenuto applicabile il metodo dei S. E., e ne ha eseguiti alcuni in corrispondenza dei fori di sonda esistenti. E opportuno, ora, riportare integralmente quanto la C.G. G. ha scritto nella relazione già citata.

"L'esame dei diagrammi mostra che gli strati della S.S. resistente si differenziano nettamente dalla copertura argillosa conduttrice; in realtà le curve rientrano nel tipo di quelle a due terreni: ricoprimento conduttore e substrato resistente.

L'interpretazione però è complicata da due difficoltà:

1. - La resistività della copertura argillosa varia sia latelalmente e sia in profondità e ciò rende più delicato il confronto dei diagrammi fra loro; la resistività è, in generale, più elevata in profondità (3,5-1 ohm) che in superficie, dove può discendere al disotto di $2 \mathrm{ohm}$.

2. - La S. S. non è un substrato resistente indefinito, bensi soltanto uno strato di spessore finito riposante sul Tortoniano molto conduttore; benchè le lunghezze di linea adottate siano troppo corte per raggiungere la discesa finale della curva, l'influenza di tale strato conduttore si fa sentire diminuendo il valore della resistività apparente sul ramo di curva corrispondente alla S. S., e tanto maggiormente quanto più la S. S. è profonda.

Per queste ragioni, questi S. E.-campione sono, nella maggior parte, poco utilizzabili per l'interpretazione dei diagrammi, poiché essi corrispondono a casi in cui la S. S. è poco profonda. Inoltre, i sondaggi Mintina 13 e Capreria 21 . non hanno raggiunto il tetto della S. S. e non forniscono quindi che un limite inferiore della sua profondità ".

Poiché la resistività della copertura argillosa varia sia latelalmente che in profonditi, viene a mancare uno dei presupposti fondamentali 
della teoria dei S. E., cioe la omogeneità degli strati: per conseguenza la stima della profondità del substrato diventa del tutto aleatoria.

I diagrammi di taratura appartengono tutti al tipo di un complesso a due strati con il superiore a resistività minore dell'inferiore, il che rontraddice alla realtà dei fatti, perchè si tratta invece di un complesso a tre strati con quello intermedio (la serie gessoso solfifera) ad alta resistività, di potenza finita e non molto grande, racchiuso fra due strati a resistivitì minore.

E vero che la conduttivitì del substrato (e della copertura, aggiungo io) fa abbassare la resistività apparente nel ramo di curva corrispondente alla serie gessoso solfifera, ma non può farla abbassare fino al punto da uguagliare quella del substrato. Laffermazione che le lunghezze di linea adottate non sono state sufficientemente lunghe per raggiungere la discesa finale della curva relativa al substrato conduttore non può essere accettata come valida.

Consideriamo infatti il S. E. n. 12 fatto in vicinanza del foro di sonda Mintina 12. E stata attraversata la seguente successione: fino a $105 \mathrm{~m}$ formazione di copertura con resistività variabile fra 2 e $4 \mathrm{ohm}$; fino a $\mathbf{1 7 0} \mathrm{m}$ serie gessoso solfifera, la cui resistività è compresa fra $\mathbf{4 5}$ e $70 \mathrm{ohm}$; fino a $178 \mathrm{~m}$ tripoli e argille molto conduttrici. In questo caso la lunghezza della linea di erogazione della corrente è stata di $1000 \mathrm{~m}$, e perciò la profondità di investigazione è di $500 \mathrm{~m}$; la curva ottenuta ̀̀ quindi relativa anche a $230 \mathrm{~m}$ di terreno argilloso molto conduttivo. Lo strato intermedio resistente influenza, a sua volta, la resistività apparente del substrato sottostante, e perciò all'inizio del tratto di curva relativo al substrato conduttivo, si avranno valori della resistività apparente superiori a quello della resistività propria del substrato; ma, con l'aumentare della profondità di investigazione, il valore della resistività apparente si avvicinerà asintoticamente alla resistività propria del substrato, ammesso che esso si estenda indefinitamente verso il basso.

Nel caso in esame la discesa della curva dovrebbe avere inizio intorno all'ascissa 200, invece risale ripidamente fino all'ascissa 350 e poi più dolcemente fino al termine (Fig. 4). Lo stesso ragionamento può essere fatto per i rimanenti S. E. -- E chiaro, dunque, che la forma dei diagrammi fa supporre l'esistenza di altri fattori disturbanti.

In base ai risultati di questi $\mathrm{S}$. E.-campione, sarebbe stato logico pronunciarsi per l'inapplicabilità del metodo in questo bacino, ma la C. G. G., fidando sulla sua più che trentennale esperienza, ha creduto opportuno passare alla fase applicativa, con una rete di S. E. con densità media di 5 per Kmq. 
La quasi totalitì dei diagrammi ò risultata del tipo riferibile ad un complesso a due strati, come quelli di taratura. A compendio dell interpretazione dei diagrammi, la C. G. G. ha presentato una carta del tetto della serie gessoso solfifera e una tavola di sezioni, che indicano la pre-

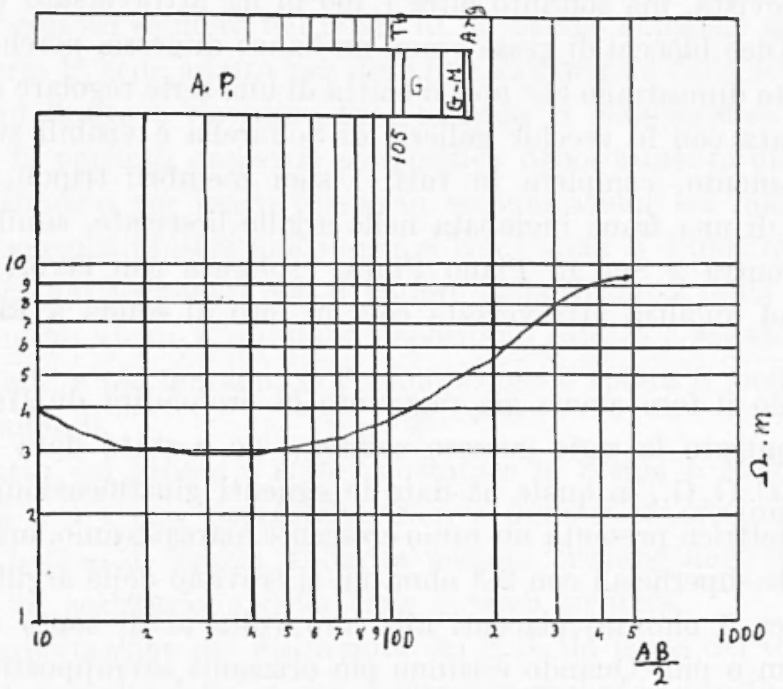

Fig. 4 - Profilo del foro di sonda 12 bis e diagramma del S.E. n. 12. $A P=$ Argille brecciate; $T b=$ Trubi; $G=$ Gessi; $G-M=$ Gesso argilloso; Arg. = Argille di fondo.

senza della serie in tutta l'estensione del bacino e di una faglia in direzione all'incirca NNO-SSE, della quale non si ha alcun indizio in superficie, che avrebbe rialzato notevolmente la parte occidentale del Bacino (Fig. 5).

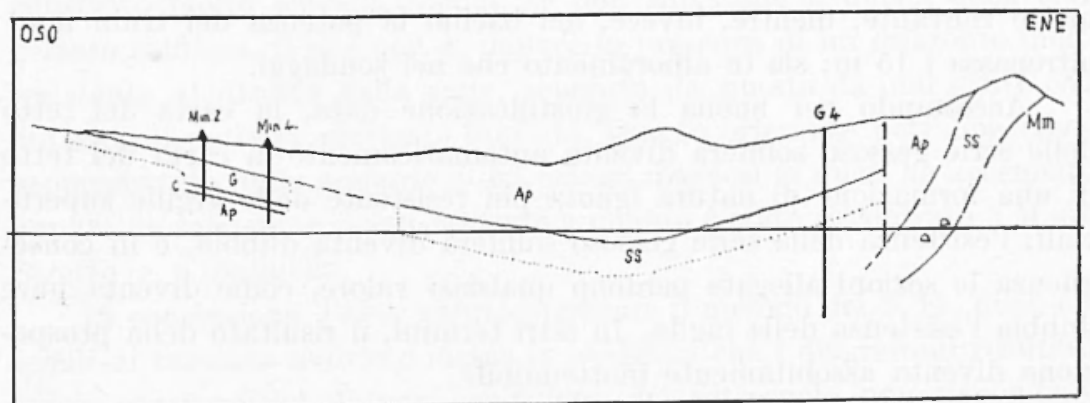

Fig. 5 - Sezione Mintina Solfarella, secondo la C. G. G.

$U m=$ Miocene marnoso; $S S=$ Serie solfifera; $C=$ calcare solfifero; $G=$ Gessi; $A p=$ Argille puddingoidi; $M i n=$ Fori di sonda esistenti. 
I'T. Z. I. ha voluto eseguire un foro di sonda di controllo, e d'accordo con la C. G. G. venne ubicato sul S. E. G4, ad ovest della presunt:t faglia, dove era prevista per il tetto della serie gessoso solfifera una profondità di $150 \mathrm{~m}$; il foro di sonda non ha incontrato la serie alla profonditic prevista, ma soltanto oltre i $100 \mathrm{~m}$ ha attraversato dei blocehi di gesso. Dico blocchi di gesso e non un banco di gesso, perchè le carote che ho visto dimostrano che non si tratta di una serie regolare come quella incontrata con le vecchie gallerie di Solfarella e visibili sul rovescio dell'affiomento, completa in tutti i suoi membri: tripoli, calcare e gesso, ma di una frana inglobata nelle argille brecciate, simile a quella che si incontra a sud di Piano Pinta, esplorata con lavori in sotterraneo, e ad un'altra attraversata con un foro di sonda a Piano Pinta nel 19 H.

Quando il foro aveva già raggiunto la profondità di $216 \mathrm{~m}$ senza avere incontrato la serie gassoso solfifera, ne ì stata data comunicazione alla C. G. G., la quale ha dato le eguenti giustificazioni: "Il diagramma elettrico presenta un ramo crescente marcatissimo, ma al disotto delle argille superficiali con $2-3 \mathrm{ohm}$. m. si trovano delle argille più resistenti (circa 5 ohm.m) giacenti alla loro volta al di sopra della S. S. con $10 \mathrm{ohm}$ o più. Quando esistono più orizzonti sovrapposti di resistività crescente, ̀̀ noto che il secondo contatto, in generale, non si manifesta nettamente nel diagramma elettrico e cio viene denominato il fenomeno della scomparsa.

In altri termini, le nostre stime di profondità si riferiscono in questo caso al tetto del primo orizzonte resistente, che può non coincidere con la S. S., come le perfordzioni recenti hanno dimostrato ".

Osservo che la giustificazione non regge, in quanto le argille piu resistenti identificabili con i trubi, avrebbero dovuto avere una potenza molto rilevante, mentre, invece, nel bacino la potenza dei trubi non oltrepassa i $15 \mathrm{~m}$; sia in affioramento che nei sondaggi.

Accettando per buona la giustificazione data, la carta del tetto delle serie gessoso solfifera diventa automaticamente la carta del tetto di una formazione di natura ignota piǹ resistente delle argille superficiali; l'esistenza della serie gessoso solfifera diventa dubbia, e in conseguenza le sezioni allegate perdono qualsiasi valore, come diventa pure dubbia l'esistenza della faglia. In altri termini, il risultato della prospezione diventa assolutamente inattendibile.

Non è necessario cercare di spiegare le cause di questo insuccesso, perchè la spiegazione è implicita nella relazione stessa e nella successiva delucidazione; può essere riassunta ricordando due dati di fatto, ammessi 
anche dalla C. G. G.: inomogeneità dello strato di copertura e forte inclinazione degli strati vicino gli affiomamenti.

A mio modo di vedere, la causa principale dell'insuccesso ra ricercata nell'aver considerato una sola ipotessi di lavoro, cioè la contiuità della serie gessoso solfifera nel fondo di un bacino supposto sinclinalico. Non è questa la sede adatta per discutere sull'origine della serie gessoso solfitera, mi limito a ricordare che, secondo la maggionanza degli studiosi, essa fa parte di una serie evaporitica depositatasi in un ambiente lagunare, rimasto per lungo tempo in commicazione col mare. Ia letteratura geologica illustra molti esempi con i quali si dimostra il variare della natura dei sedimenti con il variare della distanza dalla comunicazione col mare; vicino a questa si depositano calcare e solfato di calcio, meno solubili, e più lontano, se l'evaporazione è spinta a fondo, gli altri sali più solubi!i.

Un fatto del genere ̀̀ stato constatato in Sicilia a Monte Stretto, a Salinella e a Cozzo Sacchitello. Non i dunque inverosimile che nel fondo del bacino di Trabia Tallarita possano trovarsi degli ammassi di salgemma in sostituzione della serie gessoso solfifera.

L'interpretazione dei diagrammi dei S. S. da parte dei tecnici della C. G. G. ha risentito della scarsa conoscenza geologica diretta del bacino e delle zone viciniori. I. risultati dei fori di sonda 12 bis e 13 avrebbero dovuto suggerire, anche ammettendo la continuità della serie gressoso solfifera, la presenza di una faglia fra i due fori, tanto più che sul terreno sono visibili degli indizi di detta faglia, mentre quella supposta $i$ in contrasto con le vechie coltivazioni della Solfarella.

La mancanza di un ramo discendente nella quasi totalità dei diagrammi doveva suggerire due ipotesi: o una resistività delle argille del substrato molto elevata, vicina, se non superiore, a quella della serie gessoso solfifera, il che non è, oppure la presenza di un orizzonte molto resistente al disotto della serie, separato da questa da uno strato conduttivo, di potenza piuttosto limitata. Questo orizzonte potrebbe essere rappresentato dalle arenarie o dai calcari marnosi ai quali ho accemnato, oppure da salgemma, analogamente a quanto is stato riscontrato a Monte Stretto e a Salinella.

In conclusione, l'aver voluto applicare il metodo dei S. E., dopo che quelli di taratura avevano messo in evidenza che i diagrammi risultanti erano caratteristici di una condizione del sottosuolo non rispondente alla realta (complesso a due strati), e che risentivano della variazione orizzontali e verticali della copertura, ed, infine, l'aver tenuto presente soltanto una ipotesi di lavoro, cioè quella della continuità della serio 
gessoso solfifera, ha condotto a delle interpretazioni strutturali false, cone ì stato dimostrato senza ombra di dubbio dal foro di sonda praticato accanto al S. E. G4, e a determinazioni di profondità poco attendibili e perciò inutilizzabili in una eventuale ricerca mineraria.

Roma, Istituto Nazionale di Grofisica, Luglio 1961.

\section{RIASSUNTO}

LA. descrive due strutture solfifere siciliane: Monte Stretto (foglio 268 "Caltanissetta") e Trabia Tallarita (foglio 272 "Gela"), entrambe in provincia di Caltanissetta, sulle quali è stata eseguita una ricerca geofisica a mezzo di sondaggi elettrici, che ha dato risultati assolutamente inacrettabili.

Secondo l'A. l'insuccesso della ricerca geofisica deve essere attribuito alle condizioni di giacitura della serie gessoso solfifera, alla eterogeneità della copertura della serie stessa, molto lontane da quanto richiesto dalla teoria dei sondaggi elettrici, e principalmente al fatto di aver considerato una sola ipotesi di lavoro, cioè la continuità della serie gessoso solfifera ncl fondo di bacini supposti sinclinalici.

\section{$S U M M A R Y$}

The author describes two sulphur-structures in Sicily; Monte Stretto (leaf 268 "Caltanissetta") and Trabbia Tallarita (leaf 272 "Gela"), both in the province of Caltanissetta, on which a geophysic research was carried out by means of electrical sounding. The results are absolutely unreliable.

According to the author, the failure of the geophysic research is due to the particular position of the gypseous-sulphur series, the heterogeneous covering of the series, which is very different from what is required by the theory of electrical sounding, and particularly because only one hypothesis has been considered, that is the continuity of the gypseous-sulphur series along the bottom of supposed synclinal basins.

\section{RESUMÉ}

L'A. décrit deux structures solfifères siciliennes: Honte Stretto (feuille. 268 "Caltanissetta") et Trabia Tallarita (feuille 272 "Gela"), l'une et 
l'autre dans la province de Caltanissetta, sur les quelles il a été executée une recherche géophysique par des sondages électriques; mais elle a donne des resultats qui ne doivent pas être pris en consideration.

S'selon l'A., l'insuccès de la recherche géophysique doit être attribué aux conditions de l'arrangement de la série gypso-solfijère et à l'hétérogénéité du recouvrement de la série, tres eloignées des conditions teoriques des sondages électriques, mais surtout au fait d'avoir consideré seulement une hipotése de travail c'est à dire la continuité de la série gypso-solfifìre au fond de couvettes supposeses comme des symclinales.

\section{BIBLIOGRAFIA}

1. BaLdacci L., Deserizione geologica dell Isola di Sicilia. Mem. Deser. della Carta Geologica d'Italia, Roma, 1886.

2. Behrmann, R. B., Appunti sulla geologia della Sicilia centro-meridionale. Rap. al Min. Corporazioni, Vacuum Oil Co., in deposito presso il Serv. Geol. d'It., Roma, 1938.

3. Beneo E., Sul "Microdiapiro" di Leonforte e su quelli a nord di Gela. "Rend. Acc. Lincei ", Cl. Sc. Fis. Mat. Nat. S. VIII. 7, (1-4) Roma, 1949.

4. - Tentativo di sintesi tettonica dell'Italia peninsulare e insulare. "Boll. Serv. Geol. d'It. ", 71, Roma, (1949).

5. - Le possibilitì petrolifere della Sicilia nel quadro di una più realistica interpretazione della tettonica italiana. "Boll. Serv. Geol. d'It.", 72, Roma, 1950.

6. Compagnie Generale de GEopirysique, Etude structuarale par prospection électrique dans les regions de Caltanissetta et de Trabia - Texte italien. Relazione inedita, Paris, 1954.

7. Francavigla A., Relazione geo-mineraria sul bacino di Trabia - Tallarita, Relazione inedita, Roma, 1949.

8. - La ricerca del minerale solfifero in Sicilia. "Riv. Min. Sic.", 7, Palermo, 1951.

9. Francaviglia A. e JACOBACCI A., Appunti sul rilevamento geologico della zona sud-orientale del foglio 268 della Carta d' Italia "Caltanissetta". "Boll. Serv. Geol. d'It.", 74, f. 2, Roma, 1952.

10. Francavicilia A., Relazione sul rilevamento delle tavolette "S. Caterina Tillarmosa" $e$ "Xirbi". Relazione inedita, Roma, 1953.

11. - Relazione sul rilevamento geologieo della tavoletta "Ravanusa". Relazione inedita, Roma, 1953. 
12. J ${ }_{\triangle O U L}$ P., Recherches de soufre en Sicilie. Rapport de mission en Sicilie du 20 Juin 1952 au 14 Octobre 1952. Rapporto inedito, Paris, 1952.

13. Mantredini A., Studio geofisico della formazione gessoso-solfifera siciliana col metodo della resistività. "Boll. Serv. Geol. d'It. ", 70, Roma, 1950 .

14. Ogniben Le argille scagliose e brecciate in Sicilia. "Boll. Serv. Geol. d'It. ", 75, Roma, 1953.

15. - Le argille brecciate siciliane. Con $i$ rilievi di dettaglio di Grottacalda (Valguarnera-Enna), Passarello (Licata-Agrigento) e Zubbi (S. CataldoCaltanissetta). Mem. Ist. Geol. Paleon, Università Parlova, 18, Padova, 1954. 